\title{
On the application of playing psychology in oboe Teaching
}

\section{Yang Zhang}

Kharkov national skavorod international Normal University, Kharkov City, Kharkov state, 61002, Ukraine.

\begin{abstract}
When playing an instrument, the performance effect is closely related to the actual psychological state of the performer. The performer not only needs to have better professional skills, but also needs to have strong psychological quality, so that the performer can achieve the best state when performing an instrument.Therefore, in the process of oboe teaching, teachers need to pay attention to the use of performance psychology, improve the psychological quality of students' performance, maintain a good state, and fully integrate performance skills and personal emotions.
\end{abstract}

Keywords: performance psychology; oboe teaching; professional skills

Psychological changes will directly affect people's behavior.In the context of the deepening of education and teaching reform, the requirements for teachers' teaching have been improved. Therefore, teachers need to pay attention to the changes in teaching methods and teaching concepts, pay attention to the changes in students' psychology and attitudes in learning, and strengthen the cultivation of students' psychological quality.In this way, students can maintain good psychological quality in the face of performance activities and environmental changes, fully integrate their personal emotions into performance, and perfectly perform the movement in full emotion and solid skills ${ }^{[1]}$.

\section{The important role of playing psychology in oboe Teaching}

In the course of teaching, it can be found that in the daily rehearsal, some students are very proficient in playing repertoire and playing skills.In the performance of the content, can be played out smoothly and easily, but in the face of performance and examination, often appear distracted, forget score, stage fright, in the performance of emotional tension, it is difficult to give full play to the best performance level.Some students in rehearsal may not be satisfactory, but in large-scale performance or examination, often can easily deal with, and then in the performance to achieve better results. The occurrence of these two situations is mainly influenced by students' psychological emotions ${ }^{[2]}$.If the psychological quality of students is relatively poor, they are more vulnerable to the influence of external factors. When the external environment changes, there will be tension under the condition of reflexes.Therefore, in the case of too much psychological pressure, students' attention will be distracted, and then there will be the problem of rigid body movements. It is difficult to put all body and mind into the performance situation, and finally there will be some mistakes and many loopholes in the performance.If students have good psychological quality, they are not easily affected by external factors. When facing the audience and performance environment, they can effectively grasp their own rhythm, pay attention to self-expression, and then fully integrate their emotions into the situation, so as to achieve better results when playing.

The application of playing psychology in oboe teaching can promote the strengthening of students' psychological quality, avoid the excessive influence of external factors on their own psychology, and then reduce the emotional fluctuation and psychological change of students when playing,this can also reduce the actual psychological pressure caused by the environment, promote the stability of students' emotions, ensure that they are not affected by the outside world when playing the oboe, and then use the performance skills to perfectly perform the repertoire, fully integrate their own emotions into the performance content, and achieve relatively ideal results.

Copyright (C) 2020 Yang Zhasng

doi: 10.18282/le.v9i5.1208

This is an open-access article distributed under the terms of the Creative Commons Attribution Non-Commercial License

(http://creativecommons.org/licenses/by-nc/4.0/), which permits unrestricted non-commercial use, distribution, and reproduction in any medium, provided the original work is properly cited. 


\section{Application of performance psychology in oboe Teaching}

\subsection{Reduce students' psychological pressure}

When students perform Oboe, they are easy to be influenced by many factors, forming a greater psychological burden and pressure.For example, if students do not effectively prepare for playing repertoire, or do not have good performance skills when playing, the more prominent problem is students' psychological problems in terms of various influencing factors. The application of performance psychology in oboe teaching can play a role in psychological counseling for students, relieve the restlessness and anxiety of students during performance, ensure the formation of students' correct posture during performance, and promote the improvement of performance and reliability in

performance ${ }^{[3]}$.In the actual teaching, teachers can carry out from these aspects: first, to provide students with a pleasant and relaxed playing environment and playing space, to ensure that students are in a relatively relaxed state when playing, and then effectively integrate personal feelings and surrounding situations, and express the repertoire in a skilled and fluent way.Secondly, teachers need to have a clear understanding of their own position, put their own position in order to play a positive role in guiding students, When carrying out oboe training and oboe test, correct the mistakes of students in playing in time, and correct the mistakes, so as to promote the improvement of students' performance and performance.For example, when students' performance skills are lacking, teachers should carry out special training for students' performance skills, use multiple training methods to promote the overall quantity of students' oboe performance, realize the flexible use of performance skills, and integrate their true feelings into it ${ }^{[4]}$.If the students are not perfect in the preparation stage, the teacher should use reasonable ways to promote the students' good habits, and guide the students to check the instruments comprehensively before the formal performance.For Oboe, the sentinel is more special, so students need to prepare high-quality reed before playing, so as to avoid the overall performance quality affected by the problem of sentinel.

\subsection{Enhance the ability to cope with emergencies}

In oboe performance, students may encounter unexpected situations and some emergencies. Therefore, students need to form good adaptability, so as to ensure the smooth performance in actual performance.In the actual teaching, teachers should pay attention to the training of students' adaptability, so that students can have a clear and clear understanding of the differences between usual practice and formal performance, and effectively adjust their own mentality in the performance, fully adapt to the specific mode of performance, and avoid the problem of over tension. When teaching activities are carried out, teachers can use the way of simulation performance to carry out training. In this process, it is necessary to build performance situations for students, and then take the performance process as the basis to ensure the comfort of students in admission, appearance, clothing and seats, which should be consistent with the formal performance scene as much as possible. When playing, students should ensure the order of performance according to the order of performance ${ }^{[5]}$.At the same time, in the performance, even if there is a mistake, the performance can not be interrupted at will, so that the integrity of the performance can be fully guaranteed.At the same time, in view of the unexpected situation in the performance, the teacher can make other students speak loudly in the audience or throw some objects to the stage, so as to disturb the player's mood during the performance, enhance the students' psychological adaptability during the performance, try to ignore the impact of the surrounding environment on themselves, and put all their body and mind into the oboe performance.Due to the differences in psychological quality between students, some students will be more excited before performance, while some students will be more depressed before performance, and both of these situations will affect the performance effect.Therefore, at the end of the simulation performance, the teacher should summarize the performance of the students, guide the students in these two states to use appropriate ways to carry out psychological adjustment, maintain emotional stability during the performance, and then give full play to their own level when performing formally on the stage in the future ${ }^{[6]}$.For the more excited students, teachers can let them have a full rest, relax the nervous nerves and muscles, and supplement water and food in time to relieve the pressure and gradually calm down the mood.For some students with depression, teachers should try to avoid high difficulty and high intensity training between performances, so that students can calm their emotions in beautiful and simple phrases. The application of performance psychology in oboe teaching can promote the students"s ability to cope with various unexpected events and ensure the smooth completion of the whole performance process.

\subsection{Cultivate students' confidence}

Performance can reflect the emotional activities to a certain extent, but in actual performance, it is necessary to 
strengthen the support of logical thinking, so as to achieve effective control of the performance process ${ }^{[7]}$.In order to make students show a better effect when playing, we need to pay attention to the cultivation of students' self-confidence. The formation of self-confidence can make students stabilize their emotions and avoid the confusion of thinking and emotions in the state of lack of self-confidence.For example, in the actual teaching, teachers can give students extension encouragement, so that students can continue to play when they realize their own shortcomings.Students in the examination and performance, can not help but have concerns, if there is a mistake in the performance, it will have a bad impact on the subsequent performance ${ }^{[8]}$. Therefore, after the students finish playing, teachers need to guide the students' existing problems, ensure the integrity of the performance, and then form a complete logical thinking and psychological conception of music, avoid the confusion of thinking, and improve the overall performance effect.In order to cultivate students' self-confidence, teachers should strengthen basic training to improve students' overall proficiency in playing, and reduce their psychological anxiety through the formation of muscle memory.For the students who have a good sense of music, teachers should try to reduce the restrictions on students and let them play freely without any principle mistakes.In this process, the performance should not be limited to imitation, but also need to ensure their own independence in thinking and form their own style. At the same time, teachers should pay attention to the stimulation of students' desire to perform so as to make them continuously innovate in the performance.In addition, students should be encouraged to appreciate different styles of oboe performance, absorb the advantages of others in playing, so as to broaden their horizons, break through the limitations of thinking, give full play to their active consciousness in learning, and form a strong self-confidence in performance.At the same time, in the performance, students need to be sure to memorize the score, on the basis of mastering the score, can make the whole performance process more smooth, and use skilled skills to fully show their inner feelings.

\section{Conclusion}

In a word, the performance effect of oboe is closely related to people's psychology. In order to ensure the performance effect of students, the performance skills can be effectively developed.Teachers need to actively apply performance psychology in teaching, cultivate students' psychological quality, try to reduce students' psychological pressure in performance, realize the construction of a good environment, enhance students' self-confidence in performance, and improve students' Adaptability in performance.In this case, it can also promote the improvement of teaching quality, so that the oboe performance content can be displayed perfectly. Therefore, in the teaching of oboe, teachers should actively use performance psychology.

\section{Reference}

1. Li Wanchun. Oboe teaching and cultivation of comprehensive quality [J]. Art and technology, 2019, 32 (3): 259

2. Ju Xing. A comparative study of Chinese and Western oboe teaching methods [J]. Sichuan drama, 2018 (8): $161-164$

3. Li Wanchun. On the guiding significance of western music history to oboe Teaching [J]. Art education, 2018000 (007): p. $70-71$

4. Li bingshuai. From oral instruction to medium communication: on the teaching transformation of oboe teaching in the contemporary media environment [J]. Youth, 2017 (29): 122-123

5. Yu Jingshi. Exploration on the application of performance psychology in oboe Teaching [J]. Art technology, 2017,30 (3): 363

6. Liu haopeng. Analysis of the application of performance psychology in oboe Teaching [J]. Northern music, 2016,36 (19): 59

7. Chen Zhonghui. Analysis of the influence of oboe's mouth shape on interval accuracy -- a review of selected oboe teaching music [J]. China Education Journal, 2016 (4): 30

8. Lu Manman. Application of 3D reed modeling in oboe Performance Teaching [J]. Voice of the Yellow River, 2016 (13): 49 